\title{
Breaking groupthink:
}

Why scientific identity and norms mitigate ideological epistemology

\author{
Jay J. Van Bavel \\ Department of Psychology \\ Center for Neural Science \\ New York University \\ Diego Reinero \\ Elizabeth Harris \\ Claire E. Robertson \\ Philip Pärnamets \\ Department of Psychology \\ New York University
}

\begin{abstract}
Van Bavel, J. J., Reinero, D., Harris, E., Robertson, C. E.,\& Pärnamets, P. (in press). Breaking groupthink: Why scientific identity and norms mitigate ideological epistemology. Psychological Inquiry.
\end{abstract}

Contact information:

jay.vanbavel@nyu.edu 


\title{
Breaking groupthink: \\ Why scientific identity and norms mitigate ideological epistemology
}

\author{
"Nullius in verba (on the word of no one)" \\ - Motto of the Royal Society since 1660
}

On November 28th, 1660, the Royal Society formed to create the world's first learned society. The society adopted the motto Nullius in verba to signal their determination to withstand the domination of authority and to verify all statements by an appeal to facts and experimentation. This ethos was behind the publication of Isaac Newton's Principia Mathematica and Benjamin Franklin's famous kite experiment on electricity in the Royal Society's journal. Yet there is continued debate about the allegiances of scholars--are their scientific publications determined by their allegiance to ideological sects or do they live up to the motto and follow the evidence where it leads them?

This issue cuts to the very origins and function of science: Can scientists be trusted to conduct unbiased science? There is a growing body of papers arguing that psychological research is guided by "ideological epistemology" (see Clark \& Winegard (Clark \& Winegard), this issue; Duarte et al., 2015 for reviews). According to this account, people are innately tribal in their political dispositions and these allegiances inevitably produce groupthink and guide them away from the truth--leading to a body of flimsy or biased research. This is a serious claim and one that would likely have far-reaching implications for many fields in the social sciences, as well as branches of biology (e.g., genetics) and climatology. Yet, like any other scientific claim, it deserves careful scrutiny and rigorous analysis. In the current paper, we examine the theoretical and empirical basis for ideological epistemology in science, finding limited factual evidence for ideological bias in the published literature. 


\section{Overview}

We wholeheartedly agree with the basic premise of ideological epistemology. As we have written before, "the tribal nature of the human mind leads people to value party dogma over truth" (pg. 214; Van Bavel \& Periera, 2018). There is extensive evidence humans evolved in groups and these selective pressures have shaped us to a considerable degree (Kurzban, Tooby \& Cosmides, 2001) and we derive a sense of belonging, meaning, and security from group memberships (Tajfel \& Turner, 1979; Turner, Oakes, Haslam \& McGarty, 1994). As such, the selective pressures associated with the sociality of humans have led to a capacity to rationalize nearly any cognition in order to motivate our own behavior or to gather coalitional partners for social action (Mercier \& Sperber, 2010; von Hippel \& Trivers, 2011; Xiao, Coppin \& Van Bavel, 2015). Indeed, our powerful reasoning apparatus likely shapes reality to suit our needs in a world saturated with information and communication (cf. Pärnamets, Johansson \& Hall, 2019; Van Bavel et al., 2019). Therefore, we share the conceptual assumption that humans are groupish animals with a propensity to engage in motivated cognition to support their group identities.

Nevertheless, we believe that dispositional and situational factors could make scientists less susceptible to partisan biases--especially during their scientific decision-making. People who have a disposition to seek out and consume scientific information are far less partisan (Kahan et al., 2017), and those who possess an identity that is premised on generating accurate beliefs, like "scientists, investigative journalists, and jurors" should be more likely to hold accurate

\footnotetext{
${ }^{1}$ While we agree that most-if not all-humans possess the capacity for tribalism, we do not believe that tendency is identical across the political spectrum. For instance, recent work finds that conservatives have more parochial and liberals have more inclusive identities (Wayz, lyer, Young, Haidt, \& Graham, 2019).
} 
beliefs (Van Bavel \& Pereira, 2018). Indeed, scientists tend to require greater empirical consistency than non-scientists (Hogan \& Maglienti, 2001). Moreover, institutional tools, norms, and structures (e.g., blind peer review) are designed to mitigate against bias and increase the rigor and accuracy of scientific papers (see Merton, 1942). Although scientists may engage in partisan behavior when other identities are salient (e.g., at a political rally or college football game), their scientific identity and norms are much less likely to be active during their academic work and thus mitigate against "ideological epistemology".

Given our theoretical framework, we have conceptual and empirical critiques of ideological epistemology (Clark \& Winegard, this issue; Duarte et al., 2015). Our conceptual critique relates to how ideological epistemology ignores insights from social identity and self-categorization theory and how group memberships and concomitant norms shape behavior and cognition across contexts (see Tajfel \& Turner, 1979; Turner, Oakes, Haslam \& McGarty, 1994). We argue that the social identity approach leads to different expectations for how political ideology or partisan identity will operate in the context of a competing scientific identity. Importantly, this understanding also points towards a very different way of resolving any risks of partisan or ideological bias - one that highlights the common identity of science rather than head-counting partisan beliefs of individual scientists. Our empirical critique relates to the state of evidence for the ideological bias. We argue that most of the evidence for ideological bias has limited evidential value, and where strong studies exist, they do not support the claims of ideological epistemology in psychology.

\section{Social identities and partisan brains}


The Social Identity approach describes how people generate social identities through self-categorization and how this impacts cognition and behavior (Tajfel \& Turner, 1979; Turner, Oakes, Haslam \& McGarty, 1994; Reicher, Spears \& Postmes, 1995). Social identities are distinct from personal identity, but neither is given primacy for understanding the self. The key insight is that, depending on the situation and context, different identities (e.g., scientist, liberal, Republican, Canadian) will become salient and be used to construct the self-concept.

Across situations we have multiple potential identities which may become active. In some situations, our political and ideological leanings become salient and with them our identities as belonging to some ideological group, say Marxist or Libertarian, or some political party, like the Green party or the Conservatives. If our identities are malleable and context dependent, the critical question to ask, then, is what kind of context do we as scientists find ourselves in when doing science? Is it one that makes salient political and ideological categories or is it one that makes salient concepts related to the category of being a scientist? The answer to this question should then determine how these identities influence our decision-making,

Our thinking is grounded in the Identity-Based Model of Belief (Van Bavel \& Pereira, 2018). The model posits that whether an individual believes something is a function of the weight given to identity-based motivations versus accuracy motivations. On one hand, one's beliefs can act to fulfill group-based motivations. For example, believing a false political news story stating that your political leader won a debate against their opponent allows you to believe your group is superior to that outgroup, fulfilling status goals. Other group-based motivations include belongingness goals (wanting to feel like you belong to your group) and moral goals (wanting to believe your group is morally superior compared to outgroups). On the other hand, accuracy 
goals are the desire to hold accurate beliefs about the world. When the accuracy goals outweigh the group-based goals, people will be less likely to engage in biased processing and less likely to believe false material to be true (Bullock, Gerber, Hill \& Huber, 2015; Jakesch, Koren, Evtushenko, \& Naaman, 2018; Periera, Harris, \& Van Bavel, 2019).

The key takeaway from this model is that we can improve the accuracy of our beliefs by increasing the value of truth. Critically, we argue that our scientific identities and the institutional practices common in science do exactly that. When we are at work as scientists, our identity as a scientist is more salient (relative to the salience of our political identities). For many of sciences, our identity as scientists is deeply important to our sense of self and therefore chronically salient. One prominent feature of the identity of scientists is the norm of pursuing the truth (Nullius in verba), for the sake of their reputation and livelihood. For reputation, too much departure from the truth can lead to ostracism in the field of science. Having a reputation for conducting less rigorous science can lead to social sanctions, reduce the likelihood of finding a job or obtaining tenure, and increase the odds of being fired for fraud or p-hacking. Indeed, the invention of science centered around the development and use of novel concepts like discovery, fact, and experiment that have remained core to the scientific enterprise to this day (Wootton, 2015). Scientific training leads to the internalization and practice of these norms. As such, scientific identity can promote accuracy.

\section{Institutions and norms of science}

While emphasizing the identity of a scientist can promote the pursuit of truth, Clark \& Winegard argue that the pursuit of truth can nevertheless be "biased" by people's priors regarding their causal understanding of the social world (e.g., believing that Black Americans have a lower median income than White Americans due to societal prejudice rather than an inherent genetic 
difference). Indeed Clark \& Winegard suggest that most social scientists hold the same priors, traditionally liberal views, such that groupthink emerges. While we agree that people have different priors from reading and conducting science, we believe the norms and institutions of science are well designed--indeed, deliberately designed--to mitigate the impact of groupthink.

Groupthink occurs within a group of people in which the desire for harmony or conformity in the group results in dysfunctional decision-making outcome (Janis, 1982). This phenomenon occurs when the following conditions are in place: 1) high group cohesiveness, 2) structural faults (e.g., lack of norms regarding procedure), and 3) situational context (e.g., external threats). Janis (1982) offered nine suggestions to mitigate groupthink, including: every group member needs to be a critical evaluator of the group's decision, leaders need to be impartial and not share their personal opinion until the rest of the group is done deliberating, and there should be multiple groups thinking about the decision independently. We believe the conditions that might underlie political groupthink are rarely in place during the practice of science.

Blind peer review has been a cornerstone in science since the 1600 s and not only mitigates the problematic conditions, but even follows some of Janis' proposed suggestions to reduce groupthink. First, peer review involves multiple reviewers completing reviews independently. Having multiple independent reviewers naturally reduces "group cohesiveness", especially given that individual reviews tend to be weakly correlated (Jackson, Srinivasan, Rea, Fletcher \& Kravitz, 2011, Marsh Jayasinghe, \& Bond, 2008). Indeed, that peer-reviews are weakly correlated is the exact opposite of what you would expect to find under conditions of groupthink, suggesting that reviewers identify different strengths and weaknesses of research. Further, each reviewer critiques the submission independently, which follows one of Janis' recommendations in which each group member is a critical evaluator and another 
recommendation that multiple groups (in this case, each group being an individual) make independent decisions. Second, the editor (effectively the group leader) does not give her personal opinion to the reviewers before they review the paper, which is in line with Janis' suggestions that leaders be impartial. Third, the reviewers are anonymous. This anonymity reduces the "threat" of the reviewers' decisions regarding the manuscript, as they do not need to worry about reputational consequences that may accompany rejecting a colleague's paper.

In addition to those benefits, peer review also removes people with conflicts of interest from the process and often involves reviewers from different academic or theoretical backgrounds. The first eliminates the risk of personal bias and the second allows a critical review of the science from different perspectives and expertise. While we surely do not claim that peer-review is infallible, these features lead reviewers to highly value truth and rigorous science in their reviews and this puts pressure on scientists to engage in research and writing practices to anticipate this value of truth. Indeed, if one was hired by an organization to design institutional strategies to reduce groupthink it would be hard to beat the practice of blind peer review in the way it is implemented every day by scientists around the world.

\section{Hunches are not evidence}

Given that social science is predominantly composed of liberals, it is easy to assume a severe political bias pervades the field. Such assumptions bear out in the data wherein people predict much larger biases than the data suggest. For example, a recent analysis of 306 politically relevant abstracts from the Society for Personality and Social Psychology revealed that liberals are characterized slightly more positively than conservatives and conservatives are more often the target of explanation than liberal. Yet a separate group of academics who made predictions significantly overestimated how big these effects would be (Eitan et al., 2018). Thus, scientists 
significantly overestimate the magnitude of ideological epistemology. We suspect this gap comes from failing to realize how institutional forces and competing identities guide decision making in science and publication. (We further note that this research was conducted on conference abstracts which undergo very light peer review--where a rigorous analysis of methods is unavailable to reviewers and might further mitigate ideological epistemology.)

Of course, this does not mean other biases are absent. Some of the most notable instances of peer review bias have surrounded author and institution prestige (Merton, 1972). As people activate their academic identity, they might use author and institution prestige as markers of scientific quality and allow them to influence their reviewer decisions. For example, research conducted by high prestige institutions or well known authors were more likely to be recommended for acceptance compared to the exact same papers when no institutional information was provided (Tomkins, Zhang, \& Heavlin, 2017). Similar prestige bias findings have been found in the medical field (Okike et al., 2016; Ross et al., 2006, Garfunkle et al., 1994; Fisher et al., 1994), with evidence suggesting that single- and double-blind peer review help mitigate such prestige bias to the extent that reviewers are effectively blinded. From an identity perspective, these are precisely the types of bias that are likely to influence decisions and this work underscores the value of double-blind peer review (Tomkins et al., 2017).

Although some scientists have a hunch that ideological bias in peer-review exists, systematic evidence is scarce. And while it is possible to test the perception of ideological bias in the peer review process (Lee et al. 2013; Ceci \& Peters 1982, Benos et al. 2007), it is even more informative to know whether any such bias impacts real-world outcomes, such as whether papers are accepted or rejected, or whether papers replicate or not. These are important 
questions that require rigorous empirical research and prior work suggests that scientists cannot trust their hunches about the degree of ideological epistemology.

Clark \& Winegard (this issue) suggest that many findings in the social sciences that fail to replicate or have potentially trivially small effects are likely to be "equalitarian-friendly findings" (pg. 35). They define equalitarianism as a view held by most social scientists that demographic groups (e.g., sexes, races) do not differ genetically on socially valued traits (e.g., intelligence, criminality) and thus that any differences seen in groups arise due to societal prejudice, such that we can and should make all groups equal in society. Because equalitarianism is "strongly associated with liberalism" (Clark \& Winegard, this issue) this implies that a liberal bias is causing a lack of replicable or robust research. ${ }^{2}$ Yet this argument is tenuous, as there is no systematic evidence suggesting that liberal bias meaningfully impacts the replicability or robustness of research. In fact, the best available evidence to date suggests the contrary (Reinero et al., 2019). Thus while there may be bias in the framing of studies in social psychology, this is an empirical question that requires systematic analysis, not narrative descriptions or cherry picking.

\section{The problem with cherry-picking}

Although Clark \& Winegard claim their core argument is around biased thinking when theory or data challenge equalitarianism, they at times broaden the scope of their argument to allege a general liberal bias in the social sciences. For example, they cite a classic study which found that liberal reviewers rated a manuscript as more publishable when it described a group of leftist

\footnotetext{
${ }^{2}$ We note that liberals favoring minority out-group members is inconsistent with tribalism, unless you accept our initial premise that social identity is fluid and can be expanded to include other groups (see Van Bavel \& Cunningham, 2009).
} 
activists as healthier than a non-activist group (Abramowitz et al., 1975). However, this single study, nearly 45 years old, used a highly questionable measure of reviewer political ideology ${ }^{3}$, found mostly null results across various dependent variables (contribution to the literature, strength of writing, methodology, statistics, conclusions, total manuscript quality) and a weak effect for the "publishability" dependent variable, leading the authors themselves to conclude that "the amount of bias detected might be so slight as to be meaningless in the real world of publish or perish" (pg. 193). Indeed, one could have just as easily cited a few more recent studies that find tentative evidence of a conservative bias in peer-review against liberal-leaning diversity research (King, Avery, Hebl, \& Cortina, 2018) and gender bias research (Cislak, Formanowicz, \& Saguy, 2018). This is further evidence that the empirical case for ideological epistemology is based on weak evidence and deserves careful scrutiny.

Instead, Clark \& Winegard cite evidence of liberal bias in IRB-review in a study that found that IRB committees were slightly more likely to accept proposals to examine discrimination against women and minorities (a 49\% approval rate) than proposals to examine discrimination against white males (a 37\% approval rate), despite the fact that the proposals would theoretically treat subjects equally (Ceci et al., 1985). Leaving aside the historical reasons why such a difference might emerge, this single study had very small cell sizes (average of 8.7 IRB proposals per cell across 18 cells) and its strongest finding was actually that IRB proposals that are socially sensitive (i.e., politically relevant) are less likely to be approved than IRB proposals that are not as socially sensitive (i.e., not politically relevant). In light of the low statistical power and small effects, it is unclear how much weight to give a study with such low evidential value.

\footnotetext{
${ }^{3}$ The authors assigned reviewer ideology based on past contributions to journals or based on membership with certain APA divisions or societies, such that the manuscript shown to reviewers was presumably ideologically congruent vs. incongruent.
} 
Again, one could cite other evidence that did not find a liberal bias in academia. For instance, one study sent emails, purportedly coming from prospective applicants who had volunteered for either the Obama or McCain campaign in 2008, to directors of graduate studies from 75 top-ranked departments of social sciences and humanities (Fosse et al., 2011). They found that responses were not significantly slower or colder when responding to the student who had worked on the McCain campaign. If the findings from this unpublished paper had instead shown bias against conservatives, it's lack of publication could have been used to argue for a form of liberal bias.

Clark \& Winegard cite experimental evidence that reviewer assessments of scientific articles and pamphlets were more positive if they were consistent with the prior perspective of the reviewer (Mahony, 1977; Koehler, 1993). Yet priors should matter. Scientific consensus is reached through the accumulation of knowledge. It would be misleading to call a scientist biased if they merely acted as a rational Bayesian agent and synthesized new evidence given their priors. For example, imagine a study that found that women are less academically inclined than men. If a reviewer is surprised by and skeptical of this finding, is that bias? We would argue no: if we know that women have consistently outpaced men in earning college degrees for the past four decades (US Department of Education, 2018), that would give good empirical reason to be suspect of a single study. This same thinking pervades some of the previous studies on "liberal bias" (e.g., describing the psychology of conservatives is not necessarily biased per se in the same way that it is not biased to describe the psychology of underrepresented groups). 
When focusing on their core claim of equalitarianism, Clark \& Winegard carefully weave their arguments around ideological epistemology, consistently hedging their claims. For example, they note that "bias" against conservatives or conservative perspectives in the social sciences writ large is unlikely a serious issue (pg. 28/29) while their core argument appears to be the exact opposite. They suggest that the vast majority of social scientists hold an "equalitarian" (and predominantly politically liberal) worldview, which biases social scientists to reject empirical assertions to the contrary and ultimately distorts their understanding of humans. In fact, Clark \& Winegard argue that social scientists are biased in that they reject genetic explanations for group differences for socially valued traits that favor advantaged groups (e.g., that men are genetically smarter than women). In this nature versus nurture debate, liberal groupthink leads most social scientists to rely too much on nurture explanations and to be too quick to dismiss nature explanations (especially if group differences arise that favor an advantaged group).

Of course, this is a false dichotomy as human behavior is a product of both nature and nurture, although the exact contributions of each for various aspects of human life has been a source of intense debate for decades. Although social psychology, by definition, focuses on the social context, the field has long acknowledged that behavior is a function of both the person and the environment-elegantly captured by Lewin's equation: $B=f(P, E)$ (see Van Bavel et al., 2017 for a recent discussion). For example, sex differences have been observed in quantitative reasoning, with men outperforming women, and the researchers acknowledge the possibility for biological, sociocultural and psychosocial explanations (Reilly, Neumann, \& Andrews, 2015). Moreover, a recent survey of 335 social psychologists found that "Beliefs in evolutionary psychology were not correlated with either indicator of ideology" (pp. 151, Buss \& von Hippel, 2018). And where psychologists did tend to reject biological explanations were cases where the 
research literature has provided overwhelming evidence of sociocultural explanations (e.g., most social psychologists rejected the explanation that some ethnic groups evolved to be more or less intelligent than other ethnic groups). Thus, there is little evidence that equalitarianism plays a significant impact on the published literature.

Scientific thinking should require us to look at the totality of evidence, not simply the findings of a handful of experiment or paper. If we built an argument based on selective evidence, we could put together a narrative review arguing against vaccinations or for precognition (while ignoring the failed replications). Strong claims require larger, more representative samples of evidence. However, as Clark \& Winegard (this issue) themselves admit, much of the evidence for ideological epistemology is "limited, anecdotal, and unsystematic and therefore dismissible" (pg. 32). After reviewing the same literature, we wholeheartedly agree. While we agree that some level or forms of ideological epistemology exist in science, none of the examples presented either by Clark \& Winegard are as convincing as we would hope because they are anecdotal and cherry-picked. In the next section, we turn to a more systematic analysis on the issue of ideological epistemology in the robustness of published research.

\section{Systematic analysis of ideological epistemology}

To test the claim that political bias impacts the quality of psychological research, we analyzed 194 research findings that had also been subject to a replication attempt (Reinero et al, 2019). This analysis covered a wide and diverse range of effects, and represents, to our knowledge, the largest and most comprehensive analysis of replications in the social sciences. By drawing from other large scale replication attempts not focused on political issues, this analysis avoided cherry picking and was more representative than any other analysis of this topic. We had sets of 
coders across the political spectrum (liberal to conservative) rate the political slant of each abstract and we tested whether an association existed between the political slant of the research and its replicability, robustness and post-publication impact (citations and online discussion). Moreover, this allowed us to get an estimate of how many papers were even focused on political issues (or had an ideological slant, of any sort).

Despite the fact that our database sampled heavily from social psychology, we found that very few papers were extremely politically slanted at face value. The vast majority of findings were either not political relevant or seen as political relevant, but neither liberal nor conservative. Thus, even though the field is predominantly comprised of scientists who are politically liberal, scientists' personal political identities do not appear to manifest very strongly in their scientific publications. By proxy, these data counter the notion that psychology is replete with papers grounded in equalitarianism. This might explain why critiques of ideological epistemology require cherry picking--there are relatively few ideologically slanted papers in the field to begin with. Second, we did not find evidence that the political slant of research was associated with replicability, robustness, or post-publication impact. Thus, the claim that political beliefs "inevitably makes partisan ideological part of the publishing process" (pg. 34, Clark \& Winegard, this issue) is not well supported by our meta-analysis. Other research we described above has found that ideological bias in conference abstract is similarly a small effect (Eiten et al., 2018). We suspect that peer review helps further mitigate any potential ideological bias.

\section{Conclusion}

In sum, we agree that social identity is a fundamental aspect of human nature. When people identify with groups, this can have significant consequences for a host of behaviors. It is also 
likely that scientists share the same evolutionary heritage--and groupish tendencies--as the people we study in our experiments. However, this does not mean that scientists inevitably produce or evaluate research uncritically that aligns with their political identities. The paper by Clark \& Winegard (this issue) overlooks a few fundamental aspects of social identity. First, decades of work on social identity makes clear that we all possess multiple potential identities and the salience of these identities varies by context (Turner et al., 1994). When we are at a political rally, reading the news, or debating politics on social media, it is natural for our political identities to become more salient and drive our judgments and actions in the world. However, when we are evaluating research methods or analyzing data at our office computer, it is more likely that our identity as scientists is salient. Second, although scientists are hardly free of bias, the norms associated with any identity are key to understanding how it impacts behavior (Reicher et al., 1984). While this can elicit intergroup bias (or epistemological tribalism) in some groups, the norms of science encourage people to evaluate empirical evidence and hold one another accountable when our conclusions depart from reality. In light of these factors, we think that stereotypes about the quality of scientific work produced by prominent scholars or elite institutions is more likely to bias scientific judgments--a finding that has been rigorously documented in work on prestige bias (Tomkins et al., 2017).

We argue that scientists might be uniquely likely to evaluate empirical claims on their scientific merits when this identity is salient (see Van Bavel \& Periera, 2018). Indeed, our own analysis of the literature finds that the robustness and replicability of a wide body of research is unrelated to the ideological slant of the conclusions and that the political preferences of scientists do not closely match the political conclusions of the literature (Reinero et al., 2019). Thus, while motivated individuals can likely scour the literature and cherry-pick evidence of ideological bias, 
these claims are not grounded in large, systematic analysis of the literature. Instead, we find overwhelming evidence that it is quantifiable factors like statistical power and effect size that predict replicability in research.

In light of this evidence, we believe the natural conclusion is that we will make far more progress by shifting our focus from the politics of scientists to their identity as scientists as well as their norms and research practices. Not only are these scientific practices clearly linked to important scientific outcomes (like replicability), but they are easier to observe, quantify, and fix. Indeed, there has been a massive effort over the past half decade to recognize the pitfalls and reward solutions regarding our editorial process. Thus, it seems that changing incentive structures to more closely align to truth will do far more to improve the rigor of our field than focusing on conceptually and empirically questionable links to political ideology. Moreover, these practices will almost certainly benefit the entire field rather than applying to the narrow sliver of studies that are extremely ideological. This is why we strongly advocate for more methodological improvements and open science practices throughout the discipline. These practices also have the added benefit of opening ideological claims to careful scrutiny--which should appeal to any serious critic.

A final concern is that people appear to significantly overestimate the degree of tribal epistemology. Not only was it absent from our own meta-analysis (Reinero et al., 2019), but other work has found that scientists are prone to significantly overestimate the degree of ideological bias in conference abstracts (Eitan et al., 2018). Likewise, scientists are more likely to assume that they would not negatively evaluate papers from a conservative perspective (2.4 on a 7 -point scale where 7 = very much), while they believed that others would negatively 
evaluate them (3 on a 7-point scale). These studies suggest that liberal scientists consistently overestimate the degree of liberal bias in the literature. We also suspect that identifying political bias in the literature might be an Ideological Rorschach Test-signaling more about the ideology of the perceiver than the actual findings. Our meta-analysis found that most work is ambiguous with regard to ideological intent and, thus, people with strong ideological commitments might infer bias even when it does not exist (see Xiao et al., 2015). For instance, the litany of examples of "liberal bias" provided by Clark \& Wingard (this issue) could be construed by an extreme liberal as evidence of "conservative bias". For instance, they suggest that the relationship between climate and aggression described by the CLASH Model (Van Lange et al., 2017) is liberally biased because it does not consider genetic differences between ethnic groups. However, a liberal might suggest that the same CLASH model is conservatively biased because it ignores the history of colonialism in countries with warm climates. These are, of course, empirical questions. Our point is that we cannot trust our intuitions on this issue--perhaps because the norms and institutional practices that guide publication decisions are not fully accessible to our conscience decision-making.

In light of this prediction error, future speculation on this topic should be anchored more closely in systematic empirical evidence and less in speculation, motivated interpretations of bias, and cherry-picked evidence. We encourage scholars to continue to investigate this area using larger samples, with pre-registered analysis plans, and transparent data practices. This would allow critics to examine numerous potential sources or consequences of bias (e.g., framing, topic selection, hiring decisions, etc). Only then would we be able to trust that speculation or intuitions about epistemological tribalism among scientists are actually grounded in hard scientific evidence. Verifying such claims by an appeal to facts and experimentation would be fully in 
keeping with Nullius in verba. Until there is more convincing evidence of ideological

epistemology in science, it seems far more fruitful to focus on more objective and measurable indices of research quality to ensure our science is robust and replicable. 


\section{References}

Benos, D. J., Bashari, E., Chaves, J. M., Gaggar, A., Kapoor, N., LaFrance, M., ... \& Qadri, Y. (2007). The ups and downs of peer review. Advances in physiology education, 31(2), 145-152

Bruce, R., Chauvin, A., Trinquart, L., Ravaud, P., \& Boutron, I. (2016). Impact of interventions to improve the quality of peer review of biomedical journals: a systematic review and meta-analysis. BMC medicine, 14(1), 85.

Bullock, J. G., Gerber, A. S., Hill, S. J., \& Huber, G. A. (2013). Partisan bias in factual beliefs about politics. Quarterly Journal of Political Science 10, 519-578.

Ceci, S. J., \& Peters, D. (1984). How blind is blind review? American Psychologist, 39(12), 1491-1494. https://doi.org/10.1037/0003-066X.39.12.1491

Fisher M, Friedman SB, Strauss B. The Effects of Blinding on Acceptance of Research Papers by Peer Review. JAMA. 1994;272(2):143-146.

Garfunkel, J. M., Ulshen, M. H., Hamrick, H. J., \& Lawson, E. E. (1994). Effect of Institutional Prestige on Reviewers' Recommendations and Editorial Decisions. JAMA, 272(2), 137-138. https://doi.org/10.1001/jama.1994.03520020063017

Greengross, G., Silvia, P. J., \& Nusbaum, E. C. (2019). Sex differences in humor production ability: A meta-analysis. Journal of Research in Personality, 103886

Hogan, K., \& Maglienti, M. (2001). Comparing the epistemological underpinnings of students' and scientists' reasoning about conclusions. Journal of Research in Science Teaching: The Official Journal of the National Association for Research in Science Teaching, 38(6), 663-687

Jackson, J. L., Srinivasan, M., Rea, J., Fletcher, K. E., \& Kravitz, R. L. (2011). The validity of peer review in a general medicine journal. PloS one, 6(7), e22475.

Jakesch, Maurice and Koren, Moran and Evtushenko, Anna and Naaman, Mor, The Role of Source, Headline and Expressive Responding in Political News Evaluation (December 5, 2018). Available at SSRN: https://ssrn.com/abstract=3306403 or http://dx.doi.org/10.2139/ssrn.3306403

Janis, I. L. (1982). Groupthink: Psychological studies of policy decisions and fiascoes.

Johnson, D. J., Tress, T., Burkel, N., Taylor, C., \& Cesario, J. (2019). Officer characteristics and racial disparities in fatal officer-involved shootings. Proceedings of the National Academy of Sciences, 116(32), 15877-15882.

Kahan, D. M., Landrum, A., Carpenter, K., Helft, L., \& Hall Jamieson, K. (2017). Science curiosity and political information processing. Political Psychology, 38, 179-199

Kurzban, R., Tooby, J., \& Cosmides, L. (2001). Can race be erased? Coalitional computation and social categorization. Proceedings of the National Academy of Sciences, 98(26), 15387-15392.

Lee, C. J., Sugimoto, C. R., Zhang, G., \& Cronin, B. (2013). Bias in peer review. Journal of the American Society for Information Science and Technology, 64(1), 2-17. https://doi.org/10.1002/asi.22784

Marsh, H. W., Jayasinghe, U. W., \& Bond, N. W. (2008). Improving the peer-review process for grant 
applications: reliability, validity, bias, and generalizability. American psychologist, 63(3), 160.

Mercier, H., \& Sperber, D. (2010). Why do humans reason? Arguments for an argumentative theory. Behavioral and Brain Sciences, 34, 57-74.

Merton, Robert K. (1973) [1942], "The Normative Structure of Science", in Merton, Robert K. (ed.), The Sociology of Science: Theoretical and Empirical Investigations, Chicago: University of Chicago Press

Okike, K., Hug, K. T., Kocher, M. S., \& Leopold, S. S. (2016). Single-blind vs Double-blind Peer Review in the Setting of Author Prestige. JAMA, 316(12), 1315-1316. https://doi.org/10.1001/jama.2016.11014

Pärnamets, P., Johansson, P., \& Hall, L. (2019, September 1). Letting rationalizations out of the box. https://doi.org/10.31234/osf.io/q96g7

Reicher, S. D., Spears, R., \& Postmes, T. (1995). A social identity model of deindividuation phenomena. European review of social psychology, 6(1), 161-198.

Reinero, D. A., Wills, J. A., Brady, W. J., Mende-Siedlecki, P., Crawford, J., \& Van Bavel, J. J. (2019). Is the Political Slant of Psychology Research Related to Scientific Replicability? [Preprint].

https://doi.org/10.31234/osf.io/6k3j5

Ross, J. S., Gross, C. P., Desai, M. M., Hong, Y., Grant, A. O., Daniels, S. R., ... \& Krumholz, H. M. (2006). Effect of blinded peer review on abstract acceptance. Jama, 295(14), 1675-1680

Spier, R. (2002). The history of the peer-review process. TRENDS in Biotechnology, 20(8), 357-358.

Tajfel, H., \& Turner, J. C. (1979). An integrative theory of intergroup conflict. In W. G. Austin \& S. Worchel (Eds.), The Social Psychology of Intergroup Relations (pp. 33-47). Monterey, CA: Brooks/Cole.

Tomkins, A., Zhang, M., \& Heavlin, W. D. (2017). Reviewer bias in single- versus double-blind peer review. Proceedings of the National Academy of Sciences, 114(48), 12708-12713.

Turner, J. C., Oakes, P. J., Haslam, S. A., \& McGarty, C. (1994). Self and collective: Cognition and social context. Personality and social psychology bulletin, 20(5), 454-463.

US Department of Education. (2018). Retrieved from:

https://nces.ed.gov/programs/digest/d17/tables/dt17 318.10.asp?current=yes

von Hippel, W., \& Trivers, R. (2011). The evolution and psychology of self-deception. Behavioral and Brain Sciences, 34, 1-56.

Van Bavel, J. J., \& Pereira, A. (2018). The partisan brain: An identity-based model of political belief. Trends in cognitive sciences, 22(3), 213-224.

Wootton, D. (2015). The invention of science: a new history of the scientific revolution. Penguin UK. 